\title{
Quarks, Leptons and Unification
}

\section{P. Musset, Geneva}

(CERN)

Particles are generally divided into two classes: "hadrons" and "leptons", a division which deals mainly with their interactions. Hadrons undergo all kinds of interactions (strong, electromagnetic and weak), whereas leptons undergo only weak interactions if they are neutral, and weak plus eletromagnetic interactions if they are charged.

\section{Quarks}

The properties of the strong interactions of hadrons were difficult to clarify at first, and it is mainly since the discovery of the unitary symmetry that general laws progressively emerged. This symmetry in turn led to the concept of quarks, introduced by Gellman and Zweig in 1964 ; a hypothetical particle which has never been experimentally isolated, but which is believed to be the basic constituent of hadrons. Nucleons are supposed to be made of three quarks, and mesons to be made of a quark-antiquark pair. This simple picture was powerful enough to enable theorists to relate many of the masses, cross sections and lifetimes of particles.

Nature seems to provide a variety of quark types of which we already know several flavours (see for example, EN 9, 7/8 p. 11). It will be recalled that in addition to the "up" and "down" quarks which form the proton and neutrons, "strange" and "charmed" quarks proved to be necessary to explain the observed particles, and more recently, the existence of the new heavy meson observed at FNAL and DESY presents evidence of a new quark called "beauty". The lack of experimental evidence for the existence of free quarks has led theorists to build up theories of "confinement" but the challenge remains for experimentalists to discover direct or, at least, indirect evidence for the existence of free quarks.

Nevertheless, when dealing with complex objects undergoing complex interactions, strong interaction physics has benefitted from much clarification in the past few years, even though, neither the increasing number of fla- vours nor their hierarchy is presently explained by theory.

\section{Quarks Seen by Leptons}

The elucidation of the properties of strongly interacting particles did not come about only by the study of hadron-hadron collisions. On the contrary, it is quite remarkable that most of the experimental work was done by using lepton-hadron or even leptonlepton collisions. One of the main reasons is that hadron-hadron collisions exhibit a kind of "squared" complexity, with respect to leptonhadron collisions, since the lepton is suposed to be simple, i.e. a point-like, structureless particle. Hence, leaving aside kinematics, all the characteristics of the lepton-hadron reactions reveal the structure of the hadron. Such use of leptons to study hadrons was indeed only possible when intense beams began to be technically achievable. The advent of high energy electron accelerators and, in particular, the discovery at SLAC in 1968 of the deep inelastic processes in which electrons scatter in fact quasielastically from quarks (the point-like constituents of the nucleon), opened this field of investigation. This behaviour was confirmed by the study of the interactions of another type of lepton, the neutrinos, at CERN a few years later. The same hadron structure was indeed seen by electrons and neutrinos. This similarity between electrons and neutrinos reinforced the universal character of the description of hadrons. Furthermore it emphasized the similarity between weak and electromagnetic interactions for which there was evidence from many other directions.

\section{Unification of Weak and Electroma- gnetic Interactions}

The need for a unified description of weak and electromagnetic interactions was met with the theory of Weinberg and Salam in 1967 and 1968. This Gauge theory was proven to have the further advantage of being renormalisable, but at that time was not supported by experiment. The discovery of a new kind of weak interaction, called the neutral current, in the bubble chamber Gargamelle at CERN in 1973, gave strong support to the theory which had, as a distinctive feature, the prediction of the existence of a neutral current. Again in this domain, the lepton-hadron reactions gave the main evidence for the neutral current interaction. This is due to the fact that in other reactions, such as the hadronhadron collisions, this weak interaction is hidden by the much stronger interactions. On the other hand in lepton-lepton collisions, the processes are too rare to be easily observed. All the various lepton-hadron reactions which were observed and measured in the following years fitted the Weinberg-Salam theory, which had only one free parameter.

More recently, the theory has been successfully tested in a completely different type of interaction, i.e. the scattering of electrons on protons. The new experiment was performed at SLAC in a polarized beam, and showed the characteristic behaviour of the weak interaction: the violation of parity. By changing the electron polarization, in a mindfully random way, to avoid systematic errors, it was possible to detect a tiny asymmetry effect of the order of $10^{-4}$, which is due to the contribution of neutral currents, whilst the bulk of the scattering cross section is due to the parity-conserving electromagnetic effect. The experiment is very different from the neutrino-proton scattering in its qualitative and quantitative aspects. It is hence quite remarkable that the result agrees with the theoretical prediction using the same value for the parameter of the theory.

Note that the neutrino and the electron experiments both involve two types of neutral currents: the hadronic neutral current and the leptonic neutral current. It is obviously of high interest to investigate separately the properties of these two currents to clarify the situation.

Presently there are two instances where the experiment does not fit the 

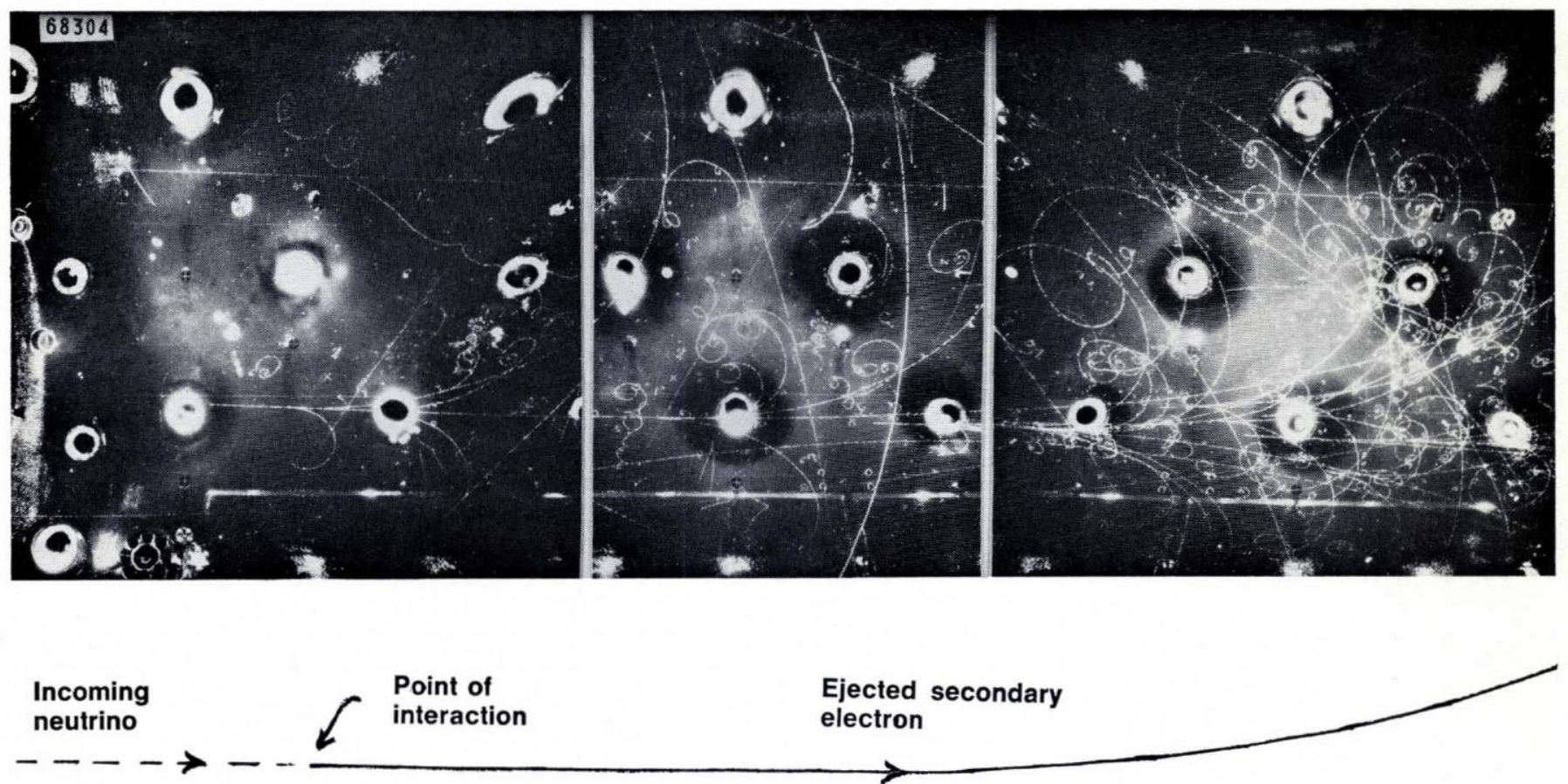

Bubble chamber photograph of one of the rare events due to a neutrino scattering on an electron, obtained in the Gargamelle experiment in the CERN neutrino beam with a flux of about $10^{13}$ neutrinos. Some 150,000 pictures, each consisting of eight frames, were analysed for this and for other reactions, and from these about 50,000 interactions of neutrinos on nucleons were seen.

The neutrino, neutral thus invisible, comes from the left and hits an atomic electron. The electron track is easily identified because the electron radiates $\gamma$ rays which are in turn converted into electron-positron pairs. This very pure reaction is of first importance in our study of the leptonic neutral current.

theory well. These two domains both involve leptons, and it is somewhat surprising that leptons which are the simplest particles should create more problems than the more complex hadrons.

\section{Leptons}

The world of leptons which, for a long time, only included electrons, muons and their corresponding neutrinos has recently been enriched by the observation at SLAC and DORIS of a heavy lepton called tau. The direct observation of its corresponding neutrino is still for the moment far from being technically feasible, but the various decay modes of the tau give rise to a neutral particle which we have every reason to identify as the tau neutrino. Also the characteristics of the decay of the tau, which are due to a charged current weak interaction, are quite similar to those of the muon.

It is probably not accidental that the number of flavours of the quark found experimentally so far is equal to the number of flavours of the lepton: there are theoretical reasons for such an equality in a unified theory.

It is in the leptonic neutral current sector that theory is faced with experimental paradoxes.

The first problem came from atomic physics. Due to a neutral-current weak interaction between the atomic electrons and the nucleons, a small parity violation effect is expected to occur in heavy atoms. This effect can be detected through the tiny rotation (about $10^{-7}$ radians) of the plane of polarization of light traversing an atomic vapour. For three years now, as reported at the EGAS Conference, two experiments, one at Oxford and the other at Seattle made in bismuth vapour and in different conditions, have failed to detect any parity violation, at a level much lower than predicted by theory. Nevertheless a recent experiment made at Novosibirsk showed an effect at the level predicted by theory. Further measurements are obviously needed. The second place in which theory faces problems is a recent result obtained on the very rare reaction of neutrinos on atomic electrons. This reaction is the only one in which only leptonic neutral currents are involved. In oposition to atomic experiments, a rate higher than that predicted by theory was observed in the Gargamelle experiment at the new
Super Proton Synchrotron at CERN. The number of events observed is ten, and the hypothesis of a statistical fluctuation will require more data to be completely eliminated. If the high rate observed is indeed a genuine effect, one has possibly seen a new phenomenon, likely to be a new neutral current which would be specific to leptons.

Both the very difficult atomic experiments and the very scarce neutrinoelectron reactions will continue to be the subject of intense investigations in the year to come. There is no doubt about the large degree of reality of the unified theory in its simplest form. If it turns out not to describe exactly the world in every detail, there are numerous reasons to believe that it would in any case be the basic approach. At the same time, the exact relation between the lepton world and the quark world still remains to be understood; variations or additions to the simplest theory have to be the subject of further investigations both in the quark and in the leptonic sectors in the near future. Later on, the hunt for new quark flavours and for new lepton flavours will continue with future accelerators. 